JJMLL

\title{
Acquisition des Collocations en Classe de Français Langue Etrangère dans les Universités Jordaniennes
}

\author{
Narjes Ennasser * \\ Département de français, Université de Jordanie, Jordanie
}

Received on: 1-7-2020

Accepted on: 1-12-2020

\section{Résumé}

La présente étude vise à évaluer les stratégies d'apprentissage et d'acquisition de collocations françaises par les étudiants des universités jordaniennes. A cette fin, un test de 30 phrases contenant 30 collocations incomplètes a été distribué auprès de 60 étudiants du niveau des troisième et quatrième années ; ils devaient compléter ces collocations en choisissant le mot approprié parmi 4 choix proposés. L'analyse des réponses des étudiants a révélé une certaine confusion au niveau des contraintes syntaxiques d'association des collocations, une influence de la langue maternelle, l'arabe, sur le choix du collocatif et une confusion au niveau des synonymes français. Par conséquent, nous proposons un enseignement insistant sur les contraintes syntaxiques et lexicales des collocations françaises, la comparaison avec les collocations arabes de même sens et même emploi et, enfin, l'introduction des dictionnaires électroniques de cooccurrence.

Mots-clés : Collocations, Contraintes, Acquisition, Evaluation, Enseignement, Français Langue Etrangère.

\section{Acquisition of Collocations by Students of French as a Foreign Language at Jordanian Universities}

\begin{abstract}
This research aimed at evaluating learning strategies of French collocations employed by Jordanian university students. For this purpose, a test of 30 incomplete French collocations has been distributed to 60 junior and senior BA students majoring in French Language and literature. The participants were asked to complete the collocations with the appropriate words to choose from four different options. Findings of the study revealed some confusion at the level of French synonyms, a lack of knowledge concerning syntactic constraints and interference from Arabic. Recommendations of the study call for the need to enhance knowledge of syntactic and lexical constraints of French collocations as well as an intensive training practice on comparing and contrasting Arabic/French collocations having similar meaning and usage through electronic dictionaries especially those dealing with collocations.
\end{abstract}

Keywords: Collocations, Constraints, Acquisition, Evaluation, Teaching, French as a Foreign Language.

\footnotetext{
๑ 2021 JJMLL Publishers/Yarmouk University. All Rights Reserved.

* Doi: https://doi.org/10.47012/jjmll.13.4.7

* Corresponding Author: ennasser@ju.edu.jo
} 


\section{Introduction}

La connaissance du vocabulaire a toujours été considérée comme une composante essentielle pour la maîtrise de la langue étrangère. Des chercheurs comme Arnaud et Béjoint (1992) affirment qu'il y a une relation étroite entre le niveau de compréhension et la connaissance du vocabulaire; Wilkins (1972) souligne que sans connaissance de la grammaire, on véhicule peu de choses mais que sans vocabulaire on ne véhicule rien du tout. Ainsi, on s'intéresse de plus en plus aux facteurs qui favorisent l'acquisition du vocabulaire. Les recherches ont montré l'importance des relations lexicales comme la synonymie, l'antonymie, etc. dans la construction du discours et la cohésion du texte. En effet, un examen minutieux de la formation de ces séquences aux niveaux lexical et syntaxique permet de mieux comprendre le système de la langue. Ellis $(1996,93)$ considère que la maîtrise des séquences polylexicales est fondamentale pour l'acquisition aussi bien de la langue maternelle que de la langue étrangère : "The attainment of fluent comprehension and production in both native (L1) and second (L2) languages involves the acquisition of memorized sequences of language ». Nous considérons, par conséquent, que la connaissance des syntagmes collocationnels revêt une importance particulière pour l'apprenant de la langue étrangère car elles sont très fréquentes en langue écrite comme en langue orale.

\section{Définition des syntagmes collocationnels}

Selon Neveu (2004) cité par Mejri (2005) : « La collocation est une cooccurrence conventionnelle, résultant d'une forte contrainte sémantique de sélection qui se manifeste dans la valence d'une unité lexicale, et qui a pour effet de restreindre la compatibilité des mots avec l'unité en question. La notion sert principalement à décrire les assemblages lexicaux habituels, autrement dit ceux qui sont entérinés par l'usage ».

Le terme collocation sera utilisé dans la présente recherche pour se référer à une séquence polylexicale qui est saisie comme une unité linguistique. En réalité, les linguistes ne sont pas d'accord sur la définition de la collocation. Leech (1974) propose une définition détaillée expliquant que la signification de la collocation dépend du sens qu'un mot acquiert selon les sens des mots qui y sont associés. Par exemple, l'adjectif bonne n'a pas le même sens dans les trois occurrences suivantes : bonne décision, bonne journée, قرار سليم، نهار جميل ، مواطنة صالحة : bonne citoyenne et sera rendu en arabe par trois adjectifs différents

Clas (1994, 576-577) explique que l'aptitude qu'ont les mots à se joindre aux autres mots est limitée, mais qu'elle est souvent imposée. Il donne l'exemple de la demande d'informations : on peut interroger, demander des renseignements ou poser des questions. On ne peut en aucun cas dire : demander une question comme l'anglais ask a question. Il cite Mel'cuk (1984), qui parle de «cooccurrence lexicale restreinte». Les mots ainsi associés montrent une affinité entre eux qui limite leurs possibilités combinatoires, éliminant certaines autres combinaisons et restreignant le nombre de sens possibles. La collocation se distingue de la combinaison libre par cette restriction, et de la combinaison figée (phrasème) par sa caractéristique de transparence. Il poursuit : « La collocation est encore une unité de la langue et, répétons-le, doit donc être enseignée et notée dans les dictionnaires et non de la parole, au sens saussurien des mots, tout comme le phrasème, mais à la différence de ce dernier, la collocation est toujours 
Acquisition des Collocations en Classe de Français Langue

Etrangère dans les Universités Jordaniennes

transparente : le sens global est déductible des unités de composition, l'un caractérisant l'autre ». Si joie débordante est une collocation signifiant une grande joie, s'en donner à cœur joie est un phrasème. Il y a également beaucoup de mots qui ne s'associent qu'avec un nombre très limité d'autres mots : perpétrer un crime, commettre une faute, émettre une protestation, porter plainte, prononcer un discours, proférer une injure, effectuer une opération, toucher un traitement, etc.

Clas propose la classification suivante :

1-verbe et nom, où le verbe a un contenu sémantique très général, sémantiquement proche du verbe faire, comme prononcer un discours.

2-nom et adjectif, comme, rude épreuve, marque distinctive.

3-adverbe et adjectif, comme, vachement bon.

4-verbe et adverbe, comme boire goulûment.

5-nom (sujet) et verbe, comme la cloche sonne, le chat miaule.

6-marquage de la quantité (unité ou collectif) du nom, comme essaim d'abeilles, troupeau de vaches, pincée de sel, barre de chocolat.

Très souvent, la combinabilité est importante et ne se dégage réellement ou n'apparaît pertinente que par comparaison avec d'autres langues. Selon Sfar (2010), la traduction de la collocation d'une langue L1 à une langue L2 se heurte à un grand nombre de problèmes. D'abord, ce qui peut être considéré comme collocationnel dans une langue ne l'est pas forcément dans l'autre. Ainsi, les équivalents en arabe de soleil شمس حارقة ، حرارة ساحقة : Alomb ne sont pas considérés comme des collocations

Ensuite, se pose le problème de la forme du correspondant en L2. Une collocation peut être traduite par une autre collocation comme dans :

poser une question طرح سؤالا

أدخل تعديلات apporter des modifications

Les éléments qui constituent une séquence collocative n'obéissent à aucune logique autre que l'usage. Ainsi, la traduction du collocatif peut être à l'origine d'une désambiguisation de la collocation en question dans la langue source. Sfar $(2010,164)$ donne l'exemple de l'adjectif accablant :

Charge accablante et preuve accablante

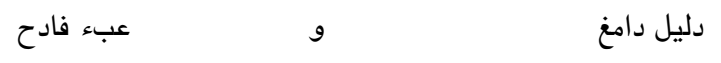

Tutin $(2005,33)$ définit les collocations comme des expressions linguistiques bi-partites, récurrentes dans la langue comme essuyer un échec ou peur bleue, dont la base (par exemple, échec dans essuyer un échec) conserve son sens premier, alors que l'autre élément, appelé collocatif (essuyer dans essuyer un échec), bien que souvent transparent, est difficilement prédictible.

Sur le plan sémantique, on peut caractériser les collocations selon le degré de figement des collocatifs (Tutin et Grossman 2002) :

1-collocations opaques (type peur bleue) lorsque l'interprétation du collocatif n'est ni transparente, ni prédictible. 
2-collocations transparentes (type beau comme un astre) où le collocatif est interprétable, mais non prédictible.

3-collocations régulières (type essuyer un échec) lorsque l'association est interprétable et d'une certaine manière prédictible, à l'aide de restrictions de sélection sémantique fines, parfois difficiles à mettre en évidence.

\section{Enseignement du vocabulaire et de la phraséologie en FLE}

Dans le Cadre Européen Commun de Référence pour les Langues (CECRL 2001, 87), la compétence lexicale est définie comme «la connaissance à utiliser le vocabulaire d'une langue qui se compose 1d'éléments lexicaux et 2-d'éléments grammaticaux ». Cavalla (2009) indique que les auteurs de ce cadre recommandent l'enseignement de la phraséologie quasiment au même titre que le reste du lexique ; elle devrait être introduite à partir du niveau $\mathrm{B}$ et être maîtrisée à la fin des niveaux $\mathrm{C}$. L'auteure précise que la problématique liée à la phraséologie est apparue intéressante dans l'enseignement d'une langue étrangère en raison de deux constats. D'abord, les apprenants non natifs font des erreurs sur ces structures ; soit ils forment des phrases complexes à la place d'une formule figée chez les natifs, soit ils utilisent un lexique compréhensible mais peu fréquent chez les natifs. Elle donne des exemples de ces erreurs comme : l'auteur continue son idée au lieu de : l'auteur poursuit son idée. Le deuxième constat est la haute fréquence de ces phraséologies dans la langue autant écrite qu'orale. Gonzalez-Rey $(2008,7)$ parle de «prêt-à-parler », de petits figements de deux mots comme rendre visite que le locuteur natif utilise abondamment sans se rendre compte de leur caractère figé et parfois métaphorique. Cavalla ajoute : «En outre, si un locuteur natif sait prévoir la fin de la collocation puisqu'il maîtrise inconsciemment le stock lexical de ces associations fréquentes, un locuteur non natif ne peut prévoir la suite d'une collocation sans la connaître en raison notamment de la métaphore utilisée. La plupart de ces expressions ne sont alors pas traduisibles d'une langue à l'autre ».

Cavalla (2016) : «Pour conclure : La phraséologie est un élément reconnu dans l'enseignement du lexique des langues étrangères mais n'apparaît pas encore systématiquement dans les manuels d'enseignement (....) Nous pouvons retenir que l'acquisition d'un mot se fait à l'aide des éléments qui l'entourent, mais surtout que retenir un élément lexical c'est mémoriser deux types d'informations : le sens

et la forme. Ceci peut paraître banal. Toutefois, l'absence de l'un (la forme des collocations) ou de l'autre (le sens des figements que finalement l'apprenant doit deviner) voire des deux dans les manuels d'enseignement du FLE est un défaut auquel il faudra remédier dans un avenir proche ».

La maîtrise des unités polylexicales, c'est-à-dire les expressions idiomatiques et les collocations, témoigne d'une compétence naturelle et presque native des apprenants de la langue étrangère. Mais les collocations sont plus faciles à enseigner et à acquérir que les expressions idiomatiques parce qu'elles ne sont pas tout à fait figées et elles admettent la substitution de l'un de leurs collocatifs ; leur sens est transparent ou au moins déductible. Néanmoins, Coulmas (1981) précise que la transparence et la facilité de compréhension de certaines collocations n'aident pas l'apprenant à les retenir car il ne fournit pas d'effort particulier et conscient pour les apprendre à moins qu'il ne lui soit demandé de faire des exercices 
Acquisition des Collocations en Classe de Français Langue

Etrangère dans les Universités Jordaniennes

dans lesquels il serait obligé d'utiliser ces collocations activement dans les communications de tous les jours. Ainsi un thé fort se traduit en arabe par : شاي ثقيل و ليس شاي قوي

\section{Objectif et méthodologie de la recherche}

La présente étude vise à évaluer la compétence communicative des apprenants du FLE, et plus particulièrement leur connaissance et leur acquisition de collocations fréquentes. Elle vise également à recenser les stratégies auxquelles recourent nos étudiants quand ils ne connaissent pas ou ne sont pas sûrs des collocations. A cette fin, un test (cf. annexe) de trente phrases françaises comprenant des collocations a été distribué auprès de soixante étudiants jordaniens inscrits pour la plupart en quatrième année ou à cheval entre la troisième et la quatrième année de la Licence en Langue et Littérature Françaises à l'université de Jordanie, qui est une université publique, un certain nombre d'étudiants d'une université privée, l'université de Pétra, ont aussi répondu à ce test. Il leur a été demandé de choisir le collocatif adéquat parmi quatre verbes ou quatre noms proposés. Il s'agit de collocations assez familières que les étudiants ont en général rencontrées dans leurs études ou dans les lectures proposées aux différents cours. Il est à noter que la majorité de nos étudiants commencent à étudier le français à l'université et le choix du niveau des troisième et quatrième années se justifie par le fait que les plans d'études de la licence en français des universités jordaniennes ne prévoient des cours de linguistique française et de traduction français-arabe qu'à partir de la troisième année ; tous les étudiants ayant participé à cette étude ont déjà suivi au moins un cours de syntaxe, un cours de linguistique et un cours de traduction. Nous nous attendons à trouver une influence de leur langue maternelle, l'arabe, sur leurs rendements. Nous allons analyser les réponses des deux points de vue quantitatif et qualitatif et, à la lumière des résultats obtenus, nous allons proposer des orientations qui pourraient aider les enseignants de FLE à sensibiliser leurs étudiants aux collocations françaises.

\section{Pourcentages de bonnes réponses}

1-Un régime politique : 28 bonnes réponses sur 60, soit 46,66\%. Cette bonne réponse est devancée par le mot système, calqué sur le mot général en arabe نظامchoisi par 32 étudiants, soit à un pourcentage de $53,33 \%$.

2-Sous le haut patronage : 24 bonnes réponses sur 60, soit 40\%, ce choix est suivi par supervision.

3-Adopter une résolution : 44 bonnes réponses sur 60, soit 73,33\%.

4-Assumer ses responsabilités : 34 bonnes réponses sur 60, soit 56,66\%.

5-Essuyer un échec : faible pourcentage de bonnes réponses, 10 sur 60, soit 16,66\%.

6-Porter plainte : 46 bonnes réponses sur 60 , soit 76,66\%

7-Faire la grève : 46 bonnes réponses sur 60 , soit 76,66\%.

8-Porter atteinte à : faible pourcentage de bonnes réponses, 8 sur 60 , soit $13,33 \%$.

9-Effet rétroactif : faible pourcentage de bonnes réponses, 18 sur 60, soit 30\%.

10-Carte d'identité : 54 bonnes réponses sur 60, soit $90 \%$. 
11-Campagne de prévention : 42 bonnes réponses sur 60, soit 70\%.

12-Le service après vente : 30 bonnes réponses sur 60 , soit $50 \%$.

13-Ceinture de sécurité : 46 bonnes réponses sur 60, soit 76,66\%.

14-Prendre des précautions : 52 bonnes réponses sur 60, soit $86,66 \%$.

15-Prendre des initiatives : 40 bonnes réponses sur 60 , soit $66,66 \%$.

16-Avoir raison. : 46 bonnes réponses sur 60 , soit 76,66\%.

17-Le taux d'intérêt : 34 bonnes réponses sur 60, soit 56,66\%.

18-L'offre et la demande : 38 bonnes réponses sur 60, soit 63,33\%.

19- Mettre à jour : 40 bonnes réponses sur 60, soit 66,66\%.

20-Rendre un service : faible pourcentage de bonnes réponses, 12 bonnes réponses sur 60, soit $20 \%$ et une يقدم خدمة influence de l'arabe avec offrir calqué sur l'arabe

21-Un coup de main : 54 bonnes réponses sur 60, soit $90 \%$.

22-Rendre hommage : faible pourcentage de bonnes réponses, 12 sur 60, soit $20 \%$.

23-Faire des efforts : 46 bonnes réponses sur 60, soit 76,66\%.

24- Mettre au courant : faible pourcentage de bonnes réponses, 16 sur 60, soit 26,66\%.

25-Porter à la connaissance de : très faible pourcentage de bonnes réponses, 6 sur 60 ,

Soit $10 \%$.

26-Jeter la lumière sur quelque chose: 30 bonnes réponses sur 60, soit $50 \%$.

27-Se mettre en colère : 38 bonnes réponses sur 60, soit 63,33\%.

28-La guerre éclata : 40 bonnes réponses sur 60, soit 66,66\%.

29-Mettre la table : faible pourcentage de bonnes réponses, 12 sur 60, soit 20\%, avec l'influence de l'arabe ranger, calqué sur ي̀ à 38\%.

30-Voir tout en noir : 40 bonnes réponses sur 60, soit 66,66\%.

\section{Analyse des réponses des étudiants}

Comme professeur de linguistique et de traduction, nous avons très souvent remarqué que les étudiants rencontrent beaucoup de difficultés à trouver les bons collocatifs français. Ils sont influencés par l'arabe et ils rendent des collocations approximatives.

Les collocations rendues correctement sont pour la plupart celles que nos étudiants ont déjà rencontrées dans leurs cours de linguistique ou de traduction ou celles qui sont similaires en arabe comme le service après- vente, la carte d'identité, la ceinture de sécurité, avoir raison, prendre des précautions ou prendre une initiative. Nous tenons dans nos cours à attirer l'attention de nos étudiants sur les différences entre l'arabe et le français, notamment en ce qui concerne les contraintes du choix du collocatif, comme porter plainte et يرفع دعوة

Parmi les réponses erronées, nous trouvons un grand nombre de calques de l'arabe, comme ranger la table calqué sur يرتب الطاولةau lieu de mettre la table et offrir un service calqué suraقدم خدمة au lieu de rendre un service. Le cas de la collocation rendre hommage attire l'attention car les réponses se répartissent 
Acquisition des Collocations en Classe de Français Langue

Etrangère dans les Universités Jordaniennes

à peu près également entre les quatre verbes proposés avec 16 bonnes réponses, 16 pour le verbe attribuer, 14 pour le verbe accorder et 14 pour le verbe support général faire ; donc, quand ils hésitent, nos étudiants recourent au verbe faire ou à un hypéronyme comme débuter au lieu d'éclater quand il s'agit de la collocation la guerre éclata. Ou encore se faire en colère au lieu de se mettre en colère. Les collocations laissées sans réponses ne sont pas nombreuses (22 sur 1800). Nous remarquons néanmoins qu'un nombre non-négligeable de nos étudiants ne sont pas conscients des contraintes syntaxiques et lexicales; ainsi nous trouvons des réponses erronées telles que : donner à la connaissance au lieu de porter à la connaissance et informer au courant au lieu de mettre au courant, montrer la lumière sur un sujet au lieu de jeter la lumière

Nous avons également noté une confusion entre des synonymes ou des mots du même champ lexical comme régime et système, résultat rétroactif au lieu d'effet rétroactif. A ce sujet, Xiao et Girani (2018, 241) parlent de fonction lexicale qui associe à chaque relation base/collocatif un sens très général et un rôle syntaxique profond. Ainsi, il est acceptable de dire «promouvoir une politique », ou bien «promouvoir la recherche » mais pas «promouvoir le développement », bien qu'ils aient la même structure et le même genre de base. L'apprenant doit donc tenir compte de cette restriction quand il choisit un collocatif pour la base car l'accent sera mis sur la fonction lexicale qu'il veut réaliser.

Concernant les collocations comprenant des noms de couleurs, comme voir tout en noir, nous avons noté autant de réponses correctes que de réponses erronées avec voir tout en rouge; nous ne pouvons pas savoir à ce stade s'il s'agit d'une confusion au niveau du sens de la collocation voir rouge, qui signifie être en colère ou au niveau syntaxique car les étudiants ne se rendent pas compte que voir tout en rouge n'existe pas et que le sens voulu est bien voir tout en noir, à savoir être déprimé.

Il semblerait que les étudiants sont influencés par leur langue maternelle, plus que par l'apprentissage de ces collocations et des contraintes fixées par l'usage du français. Même si les collocations sont utilisées dans les manuels de FLE, les étudiants ne s'en rendent pas toujours compte ni ne les retiennent. Il faudrait donc que l'acquisition de ces structures soit ciblée, c'est-à-dire que l'enseignant devrait attirer leur attention sur ces constructions particulières du français en leur donnant des exercices en cours de linguistique ou de traduction et en les comparant avec leurs équivalents arabes de façon à montrer que les collocations ne sont pas nécessairement les mêmes dans les deux langues. D'autres erreurs révèlent une confusion entre les synonymes ou des mots proches appartenant au même champ lexical, comme régime et système, faire et effectuer, attribuer et accorder. Leurs sens sont proches par rapport à certaines collocations mais un seul est consacré par l'usage, d'où la confusion pour un apprenant étranger n'ayant pas beaucoup de lectures ou d'heures d'écoute du français. Il est à noter à ce propos que la plupart de nos étudiants commencent à apprendre le français à l'université et seraient censés atteindre, à la fin de leurs études universitaires, le niveau B1 du Cadre Européen Commun de Référence pour les Langues.

\section{Implications pour la classe de FLE}

Comme le montrent les pourcentages de bonnes réponses et l'analyse, les erreurs sont dues soit à l'influence de la langue maternelle, à savoir l'arabe, ou au manque de connaissance de la nature de ces expressions, notamment des contraintes dans le choix du collocatif. Il est donc indispensable d'attirer 
l'attention des apprenants sur les types et les particularités des collocations. Brown (1994) propose une stratégie active pour les apprenants de niveau avancé car la compétence d'associer les mots est un critère important pour évaluer l'étendue des connaissances du vocabulaire chez les apprenants. Binon et Verlinde (2004) considèrent que la compétence combinatoire est la clé de voûte de la compétence de communication et préconisent de lier l'enseignement de la grammaire avec celui du lexique ; il est ainsi important d'attirer l'attention des apprenants sur les constructions syntaxiques dans lesquelles entre un mot. Ils précisent que la compétence textuelle et la compétence lexicale sont intimement liées: «Dans les exercices lexicologiques on devrait retrouver les mêmes réseaux sémantiques, les mêmes contextes, les mêmes schémas discursifs, les mêmes discours que lors de la lecture ». Ils insistent aussi sur la dimension culturelle : l'enseignant devrait expliciter les implicites culturels car les apprenants étrangers n'ont pas l'intuition des locuteurs natifs. Ils soulignent enfin l'autonomie de l'apprentissage, d'où la nécessité d'initier les apprenants à l'usage des dictionnaires.

Il serait ainsi indispensable de proposer aux apprenants des supports authentiques comprenant des collocations en contexte habituel de communication dès le début de leur parcours d'apprentissage. Ces supports pourraient être oraux ou écrits. L'enseignant devrait sensibiliser les apprenants à l'emploi de ces collocations en leur donnant des exercices variés. Nous en donnons quelques exemples que nous avons pratiqués dans nos classes :

1-Complétez les collocations qui suivent d'après leur emploi dans le texte que vous avez lu ou écouté.

2- Complétez les phrases qui suivent avec les collocations que vous avez rencontrées dans le texte lu ou écouté.

3- Ecrivez la collocation qui correspond à la paraphrase qui suit.

4-Donnez l'équivalent français des collocations arabes qui suivent.

5-En écoutant le texte, notez les collocations se rapportant aux couleurs ou aux parties du corps ou autres.

6-Cherchez dans un dictionnaire toutes les collocations dans lesquelles entre le verbe porter : porter plainte,

porter des fruits, porter atteinte, etc. ou le verbe prendre, prendre une décision, prendre des précautions, prendre l'initiative, etc.

Quel est le rôle des dictionnaires spécialisés dans l'apprentissage de la phraséologie?

Tremblay (2014) et Smadi (2019) font remarquer que les locutions et les collocations ne sont pas systématiquement répertoriées ni décrites comme telles dans les dictionnaires courants monolingues ou bilingues. On les trouve plutôt dans les sections réservées aux exemples et toutes les collocations possiblement contrôlées par un mot n'y figurent pas. C'est pourquoi il faut recourir aux dictionnaires spécialisés des collocations comme le Grand dictionnaire des Cooccurrences, qui répertorie les collocations associées à plus de 5000 mots ou le dictionnaire électronique Antidote qui présente les collocations associées à un mot dans une section spécifique appelée la zone cooccurrence. Ainsi, l'enseignant pourrait demander aux apprenants d'identifier les verbes qui accompagnent un mot-base de façon privilégiée en utilisant le classement syntaxique proposé par un dictionnaire électronique de collocations. 


\section{Acquisition des Collocations en Classe de Français Langue}

Etrangère dans les Universités Jordaniennes

\section{Conclusion}

Pour pouvoir évaluer la connaissance du lexique français chez nos étudiants et leur sensibilité aux collocations, nous avons distribué un test de 30 phrases contenant 30 collocations incomplètes ; le mot-base était donné et l'apprenant devait le compléter avec le collocatif approprié qu'il devait choisir parmi quatre mots proposés. L'analyse des réponses de nos étudiants montrent qu'ils ne sont pas sensibles aux contraintes syntaxiques des collocations, qu'ils confondent les synonymes et qu'ils ont tendance à tomber dans le piège du transfert négatif en calquant les collocations de l'arabe. Ainsi, il est nécessaire de les sensibiliser aux particularités syntaxiques et au lexique du français en proposant des exercices sur les collocations dans le cadre des cours de linguistique, de traduction ou de compréhension.

Cette modeste recherche a montré que les collocations sont difficiles à acquérir pour les apprenants étrangers. Les nuances des synonymes et le transfert négatif de la langue maternelle sont à l'origine des erreurs. Néanmoins, il n'est pas impossible d'exploiter ces influences pour attirer l'attention de nos étudiants sur les spécificités des collocations avec des exercices bien ciblés.

Enfin, nous préconisons d'initier nos étudiants aux dictionnaires électroniques de cooccurrences comme Antidote et Le Grand dictionnaire des cooccurrences et de conduire des recherches supplémentaires auprès d'étudiants du niveau du Master en englobant d'autres types de collocations pour recenser les facteurs qui déterminent leurs stratégies d'apprentissage de ces structures et leur sensibilité vis-à-vis des contraintes d'association. 


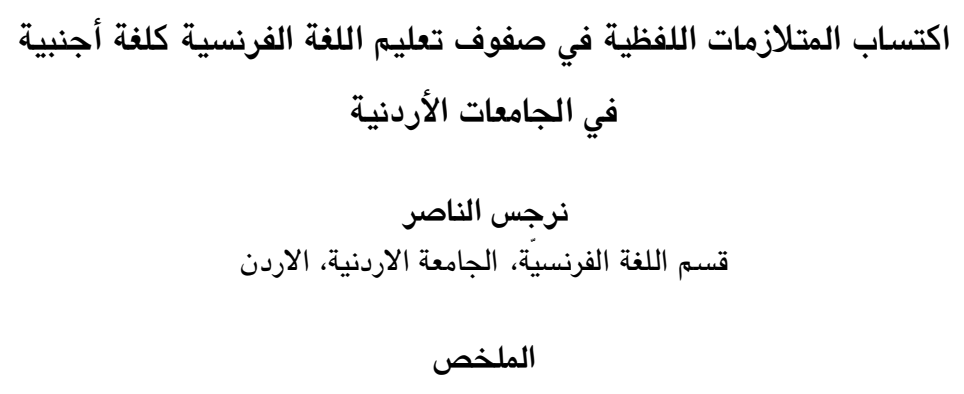




\section{Acquisition des Collocations en Classe de Français Langue}

Etrangère dans les Universités Jordaniennes

\section{Références}

Arnaud, Piere J. L. and Henri Béjoint. 1992. Vocabulary and applied linguistics. Basingstoke, Hampshire: Macmillan Academic and Professional.

Beauchesne, Maude and Kim Beauchesne. 2009. Le Grand dictionnaire des cooccurrences. Montréal, Guérin.

Binon, Jean and Serge Verlinde. 2004. L'Enseignement/Apprentissage du Vocabulaire, et la Lexicographie Pédagogique du Français sur Objectifs Spécifiques (FOS) : Le Domaine du Français des Affaires. Etudes de Linguistique Appliquée 3 (135) : 271-283

Brown, Phillip. 1994. Lexical Collocation: A Strategy for Advanced Learners. Modern English Teacher 3(2): $24-27$

Cavalla, Cristelle. 2009. "La Phraséologie en Classe de FLE” in Langues Modernes, Association des Professeurs des Langues Vivantes (APLV), consulté en ligne sur le site Hal 00699916

Cavalla, Cristelle. 2016. Synthèse HDR. consulté en ligne sur le site Hal 4/9/2020

Clas, André. 1994. Collocations et Langues de Spécialité. Meta: Journal des Traducteurs d'Interprètes 39 (4). consulté le 7/11/2018. Presses de l'Université de Montréal.

Coulmas, Florian. 1981. Conversational Routine: Explanations in Standardized Communication Situations and Prepatterened Speech. New York: Mouton Publishers.

Ellis, Nick. C. 1996. Sequences in SLA: Phonological Memory, Chunking and Points of Order. Second Language Acquisition 18: 91-126.

Conseil de l'Europe. 2001. Cadre Européen Commun de Références pour les Langues. Paris, Didier.

González -Rey, I. 2002. La Phraséologie du Français. Toulouse, Presses universitaires du Mirail.

Leech, Geoffrey. 1974. Semantics. London: Penguin.

Mejri, Salah. 2005. Figement Absolu ou Relatif: La Notion de Degré de Figement. LINX, revue des linguistes de l'université Paris X Nanterre 53 : 183-196. consulté le 7/11/2018

Mel’čuk, Igor. 1984 : Dictionnaire Explicatif et Combinatoire du Français Contemporain, vol. I, Montréal, Les presses de l'Université de Montréal.

Neveu, Frank. 2004. Dictionnaire des Sciences du Langage. Paris, Armand Colin.

Sfar, Inés. 2010. Figement et Incorporation : 1'Etablissement d'un Concordancier Bilingue (FrançaisArabe). Meta : Journal des Traducteurs et d'Interprètes 55 (1). consulté le 7/11/2018.

Smadi, Adnan. 2019. Le Traitement Lexicographique des Expressions Idiomatiques : L'Exemple de Deux Dictionnaires Bilingues Franco-Arabes. Jordan Journal of Modern Languages and Literatures (JJMLL) $11(2): 149-165$

Tutin, Agnès. 2005. Le Dictionnaire de Collocations Est-Il Indispensable?. Revue Française de Linguistique Appliquée Vol X-2.

Tutin, Agnès and Francis Grossman. 2002. Collocations Régulières Et Irrégulières: Esquisses de Typologie du Phénomène Collocatif. Revue Française de Linguistique Appliquée 7 : 7-26. 
Trémblay, Ophélie. 2014. Les Collocations : des Mots Qui Font La Paire. La poésie hors du livre 171 : 74 76. consulté le 24/10/2019

Wilkins, D.A. 1972. Linguistics in Language Teaching. Edward Arnold.

Xiao, Chen and Frédéric Girani. 2018. Typologie d'Erreurs des Collocations dans les Essais Argumentatifs et Perspectives Didactiques de l'Enseignement du Français Langue Etrangère dans les Universités. Synergies Chine 13 : 229-246. consulté le 24/10/2019.

\section{Annexe : Test d'évaluation}

\section{Complétez les phrases suivantes en choisissant les mots qui correspondent aux collocations} françaises :

1-Le politique en Jordanie est une monarchie constitutionnelle.

a-système

b-gouvernement

c-règne

d-régime

2-La cérémonie aura lieu sous le (la) haut(e) du prince héritier.

a-supervision

b-patronage

c-protection

d-direction

3-La résolution a été. à l'unanimité.

a-acceptée b-admise -adoptée d-consentie

4-Le premier ministre ses responsabilités vis-à-vis de ses citoyens.

a-assume

b-supporte

c-accepte

d-adopte

5-Il s'est présenté aux élections mais il a

un échec.

a-reçu

b-rencontré

c-essuyé

d-trouvé

6-Pierre a décidé de plainte contre le Journal du midi pour diffamation.

a-demander

b-dresser

c-porter

d-annoncer

7-Les ouvriers

la grève pour demander l'augmentation du SMIC.

a-effectuent

b-font

c-réalisent

d-accomplissent

8-Nos lois ne doivent pas

a-apporter

b-susciter atteinte aux droits de l'Homme.

9-La loi sera appliquée à

$$
\text { c- causer }
$$

d-porter

a-résultat

b- effet

rétroactif (ve)

10- A l'examen, vous devriez présenter votre carte de a-personnalité

b-identité

c-famille

d- individu.

11-Le gouvernement lance une campagne pour le (la)

a-prévoyance

b-prévention

c-guérison

des maladies infectieuses.

12-Le (la) après-vente est ouvert 24heures sur 24.

a-branche

b-section

c-service

d-division

13-On demande aux passagers d'attacher leur ceinture de

a-sûreté

b-protection

c-sécurité

d-assurance.

14-Je toujours des précautions lorsque je roule dans les rues commerçantes.

a-prends b-fais c-mets d-pose

15-Il suit toujours ce qu'on lui propose de faire ; il ne jamais d'initiative. 
Acquisition des Collocations en Classe de Français Langue

Etrangère dans les Universités Jordaniennes

a-exprime

16-Pierre avait

-droit

17-Le taux de

a-avantage b-fait

c-prend

d-décide.

de laisser tomber ce projet trop ambitieux.

b-raison

c-mérite

d-pouvoir

dans cette banque est de $10 \%$

b-bénéfice c-gain

d-intérêt.

18-Les prix des appartements dépendent beaucoup de l'offre et de la (du)

a-demande

b-besoin

c-nécessité

d-désir

19-Je vous soutiens

a-Cœur et âme

b-Corps et âme

c-cœur et esprit

d-corps et esprit

20-Tu peux me

un service? , j'ai besoin de 100 dinars.

a-donner

b-rendre

c-offrir

d-faire

21-Donne-moi un coup de. je ne peux pas faire ce travail tout seul.

a-aide

b-main

c-service

d-soutien

22-Le gouvernement français a hommage à Jacques Chirac à sa mort.

a-attribué

b-accordé

c-rendu

d-fait

23-Vous devriez plus d'efforts pour obtenir une bourse.

a-apporter

24-L'Université

b-faire

c-accorder

d-exercer

a-informe les étudiants régulièrement au courant des changements administratifs.

25-Il faut b-rend

c-porte

d-met

ces informations à la connaissance du directeur.

a-apporter

b-porter

c-rendre

d-donner

26-Cette recherche la lumière sur les difficultés d'apprentissage du français.

a-porte

b-jette

c-lance

d-montre

27-Lorsqu'il a appris que son fils avait fui de l'école, il

en colère.

a-s'est pris

b-s'est mis

c-s'est donné

d-s'est fait

28-La première guerre mondiale

en 1914.

a-débuta

b-explosa

c-éclata

d-brûla

29-Nous avons fini la cuisine et maintenant nous allons

la table.

a-mettre

b- ranger

c-faire

d-organiser

30-Quand j'ai une mauvaise note, je vois tout en

a-bleu

b-rouge

c-noir

d-blanc 